\title{
Onset of interfacial waves in the terahertz spectrum of a nanoparticle suspension
}

\author{
Alessio De Francesco, ${ }^{1,2}$ Luisa Scaccia,${ }^{3}$ Ferdinando Formisano, ${ }^{1,2}$ Eleonora Guarini $\odot,{ }^{4}$ Ubaldo Bafile $\odot,{ }^{5}$ \\ Marco Maccarini $\odot,{ }^{6}$ Ahmet Alatas $\odot,{ }^{7}$ Yong Q. Cai $\odot,{ }^{8}$ Dmytro Nykypanchuk, ${ }^{9}$ and Alessandro Cunsolo $\oplus^{8}$ \\ ${ }^{1}$ Consiglio Nazionale delle Ricerche, Istituto Officina dei Materiali, Operative Group in Grenoble (OGG) F-38042 Grenoble, France \\ ${ }^{2}$ Institut Laue-Langevin (ILL), F-38042 Grenoble, France \\ ${ }^{3}$ Dipartimento di Economia e Diritto, Università di Macerata, Via Crescimbeni 20, 62100 Macerata, Italy \\ ${ }^{4}$ Dipartimento di Fisica e Astronomia, Università di Firenze, via G. Sansone 1, I-50019 Sesto Fiorentino, Italy \\ ${ }^{5}$ Consiglio Nazionale delle Ricerche, Istituto di Fisica Applicata "Nello Carrara," via Madonna del Piano 10, \\ I-50019 Sesto Fiorentino, Italy \\ ${ }^{6}$ Université Grenoble-Alpes, CNRS, Grenoble INP, TIMC-IMAG, 38000 Grenoble, France \\ ${ }^{7}$ Argonne National Laboratory, Advanced Photon Source, P.O. Box 5000, Upton, New York 11973, USA \\ ${ }^{8}$ Brookhaven National Laboratory, National Synchrotron Light Source, NSLS II, P.O. Box 5000, Upton, New York 11973, USA \\ ${ }^{9}$ Center for Functional Nanomaterials, Brookhaven National Laboratory, Upton, New York 11973, USA
}

(Received 5 May 2020; accepted 13 July 2020; published 3 August 2020)

\begin{abstract}
We used inelastic $\mathrm{x}$-ray scattering to gain insight into the complex terahertz dynamics of a diluted Aunanoparticle suspension in glycerol. We observe that, albeit sparse, Au nanoparticles leave clear signatures on the dynamic response of the system, the main one being an additional mode propagating at the nanoparticle-glycerol interface. A Bayesian inferential analysis of the line shape reveals that such a mode, at variance with conventional acoustic modes, keeps a hydrodynamiclike behavior well beyond the continuous limit and down to subnanometer distances.
\end{abstract}

DOI: 10.1103/PhysRevE.102.022601

\section{INTRODUCTION}

Understanding acoustic propagation through inhomogeneous media is of formidable scientific relevance in many areas, including medical ultrasonography, seismology, vibration noise reduction, and nondestructive testing. From a fundamental perspective, what makes this topic especially compelling is the wide variety of phenomena happening over distances comparable to or shorter than some heterogeneity size $l$, such as an interlayer spacing, a cluster width, or a nanoparticle diameter. In a scattering experiment, the presence of a homogeneity breach might emerge whenever the exchanged wave vector $Q$ matches $2 \pi / l$. Under this condition, this broken symmetry possibly results in the onset of multiple spectral modes [1]. Colloidal suspensions in liquids are among the simplest prototypes of heterogeneous materials exhibiting a multiple excitation behavior, as suggested by Brillouin light scattering (BLS) measurements [2-6].

Although dynamic studies on liquid suspensions are well documented in the literature, they seldom cover the mesoscopic regime probed by inelastic $\mathrm{x}$-ray (IXS) and neutron scattering. In this extremely high- $Q$ window, the inequality $Q d_{c} \gg 1$ (with $d_{c}$ being the colloid diameter) holds validity even for nanometer-sized colloids, and acoustic propagation is dominated by multiple scattering. Indeed, reflections of acoustic waves at the colloid interface become more likely and prevent sound waves in the liquid from leaking into the colloid, and vice versa. Consequently, this high $Q$ regime is characterized by the coexistence of acoustic modes selectively propagating either through the solid or through the liquid, or at their interface. The latter modes are customarily referred to as Stoneley waves [7], and their presence in colloidal suspensions was documented by BLS measurements [2,4]. In addition, owing to multiple reflections, acoustic waves acquire substantial mutual dephasing, and their interference thus drastically enhances acoustic damping. Interestingly, both effects mentioned above have been observed in a recent IXS study on an aqueous suspension of $\mathrm{Au}$ nanoparticles (Au-Np) [8] and partially in Ref. [9].

This study proves that the immersion of even a sparse amount of nanoparticles (Np) in a fluid can significantly affect the terahertz acoustic propagation. Even though preliminary, this important result revitalizes the intent of controlling the terahertz acoustic properties of a medium upon structural modifications at a mesoscopic scale. Shedding further light onto this fascinating topic is one of the strongest motivations of this work.

Given these grounds, we measured the IXS spectrum of diluted suspensions of Au nanospheres in glycerol using a well-assessed Bayesian analysis which enabled the determination of the number and shape of excitations contributing to it [10]. The volume concentration was about $0.5 \%$, and $d_{c}$ was equal to either 50 or $200 \mathrm{~nm}$. These IXS measurements were jointly analyzed with previous IXS data on pure glycerol at 660 bar and ambient temperature [11].

\section{MATERIALS AND METHODS}

Measurements were executed using the Sector 30 beamline $[12,13]$ of the Advanced Photon Source (APS) at Argonne National Laboratory. The instrument was operated using the $\approx 23.7 \mathrm{keV}$ harmonic of the undulator source, which corresponds to the $\mathrm{Si}(121212)$ backscattering reflection from both 
the monochromator and energy analyzers. Spectral acquisitions covered the $3 \div 21 \mathrm{~nm}^{-1} Q$ range with a $2-\mathrm{nm}^{-1} Q$ step. The instrumental resolution function was measured through the IXS signal from a Plexiglas sample at the $Q$ of its first sharp diffraction maximum, i.e., about $10 \mathrm{~nm}^{-1}$. The resulting spectrum had a $0.8-\mathrm{meV}$, broad (half width at half maximum, HWHM) nearly Lorentzian profile, sufficient to properly resolve the relevant spectral features discussed in the remainder of this paper.

Citrate-capped Au-Np highly monodisperse in both size and shape were purchased by Nanopartz and then immersed in a matrix of $99.9 \%$ pure glycerol. According to the manufacturer specifications, the size variance was less than $2 \%$. The suspensions were embedded in 10- $\mu$ m-thick quartz capillaries having $1 \mathrm{~mm}$ outer diameter and wax-sealed on the top. The electronic noise background and empty cell scattering were found to yield a negligible contribution to the measured signal.

\section{EXPERIMENTAL RESULTS AND ANALYSIS}

Although the Au-Np are sparse, the dynamic response of the suspensions differs significantly from that of pure glycerol, as clearly shown in Fig. 1. There the spectral shapes

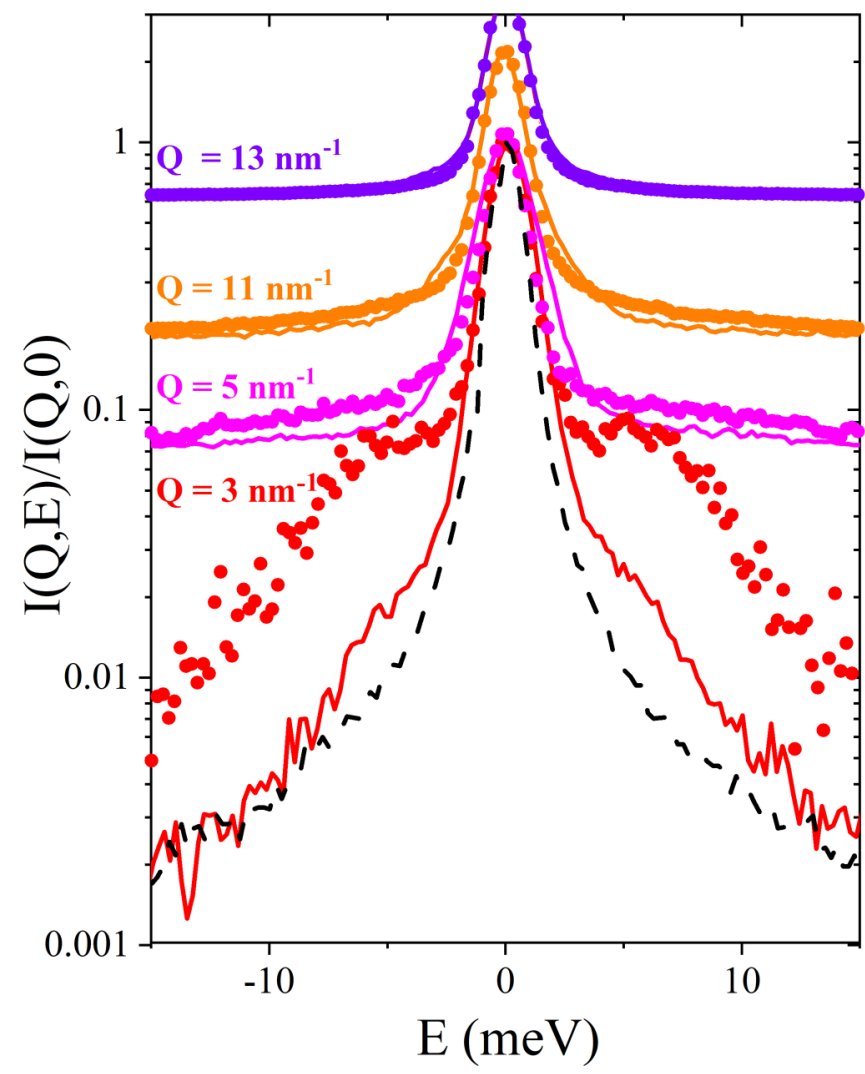

FIG. 1. Representative IXS spectra of the (200 nm sized) Au-Np suspension (solid lines) are compared with their glycerol counterparts (dots). Each suspension spectrum was collected at the $Q$ value indicated, while the corresponding spectral profile of pure glycerol was measured at $Q$ values higher by $0.5 \mathrm{~nm}^{-1}$. The lowest $Q$ spectra are also compared with the energy resolution function after a similar normalization (black dashed curve). For the sake of comparison, all line shapes are normalized to their maxima and are vertically offset for clarity. measured on the 200-nm Au-Np suspension at some representative $Q$ 's are compared with pure glycerol spectra; the latter spectra were collected in previous IXS measurements [11] carried out with the same spectrometer setup yet on a sample at a higher pressure (660 bar). Spectra from the 50-nmdiameter particles are instead reported in Fig. S1 of the Supplemental Material (SM) of this paper [14]. In Fig. 1 all curves are normalized to the respective maxima and vertically offset for clarity; furthermore, the resolution profile pertinent to the $Q=3 \mathrm{~nm}^{-1}$ spectrum is also reported for reference after similar normalization.

A few qualitative trends readily emerge even from a quick inspection of the plotted line shapes. First, at the lowest $Q$ 's the inelastic portion of the pure glycerol spectrum is dominated by broad side shoulders arising from generalized acoustic modes, whose relative amplitude seems greatly depressed upon $\mathrm{Au}-\mathrm{Np}$ immersion, consistently with what is reported in our previous IXS work on an aqueous Au-Np suspension [8]. Also, upon increasing $Q$, these side shoulders rapidly broaden and lose intensity to the extent of becoming essentially indistinguishable from the spectral background. Most importantly, let us focus on the Au-Np suspension spectra which have a central peak significantly broader than their pure glycerol counterparts; below we will discuss this aspect in further depth.

To shed some light on this difference, we performed a line-shape modeling of the measured spectra in which the IXS intensity was approximated as

$$
I(Q, E)=S(Q, E) \otimes R(Q, E)+B(E),
$$

where $E=\hbar \omega$ is the energy transferred from the probe particle to the target sample. Here the symbol " $\otimes$ " denotes the convolution operator, while $R(Q, E)$ and $B(E)$ are the instrumental resolution function and an usually mildly (linearly) energy-dependent spectral background. In Eq. (1), $S(Q, E)$ is the dynamic structure factor that can be written as $S(Q, E)=$ $K(E) S_{\text {sym }}(Q, E)$ by introducing the energy-dependent detailed balance coefficient $K(E)=\left(E / k_{B} T\right)[n(E)+1]$, with $T$ and $k_{B}$ being the temperature and the Boltzmann constant, respectively. This term accounts for the statistical population of the generic $E$ state via the Bose statistics factor $n(E)=$ $\left(e^{E / k_{B} T}-1\right)^{-1}$ and produces the correctly asymmetric spectral shape. Finally, in Eq. (1) we assumed

$$
\begin{aligned}
S_{\mathrm{sym}}(Q, E)= & A_{e}(Q) \delta(E)+L_{A_{0}, z_{0}}(Q, E) \\
& +\sum_{j=1}^{k} A_{j}(Q) D H O_{j}(Q, E),
\end{aligned}
$$

where $\delta(E)$ is the Dirac delta function accounting for the elastic peak in the spectrum having amplitude $A_{e}(Q)$ and accounting for all dynamic processes in the sample, which are too slow to be resolved by the measurement; the other terms are a quasielastic Lorentzian contribution $L_{A_{0}, z_{0}}(Q, E)$ having width $z_{0}(Q)$ (HWHM) and amplitude $A_{0}(Q)$, and $k$ inelastic contributions modeled as damped harmonic oscillator (DHO) profiles with amplitudes $A_{j}(Q)$, written as

$$
D H O_{j}(Q, E)=\frac{2}{\pi} \frac{\Omega_{j}^{2}(Q) \Gamma_{j}(Q)}{\left[E^{2}-\Omega_{j}^{2}(Q)\right]^{2}+4\left[E \Gamma_{j}(Q)\right]^{2}},
$$


where $\Omega_{j}(Q)$ and $\Gamma_{j}(Q)$ are the undamped energies and the damping coefficients of the DHO excitations. Notice that the number $k$ of $\mathrm{DHO}_{j}(Q, E)$ excitations likely to appear in the spectrum and their shape coefficients are equally treated as adjustable parameters. Finally, a Lorentzian contribution is needed to account for possible quasielastic contributions due to a structural relaxation, in analogy to what was already done in water $[8,15,16]$.

Best-fitting values of the model line shape were determined using a Bayesian inferential analysis implemented through a Markov chain Monte Carlo (MCMC) routine with reversible jump (RJ) steps. This approach can be used to probabilistically infer the joint posterior probability distribution of model parameters, which conveys important information about uncertainties of best-fitting results. Of course, the knowledge of the entire posterior distribution of each parameter also enables one to identify the best-fitting value of such a parameter with the mode of its posterior, whenever the latter, albeit not necessarily symmetric, is sharply peaked, well-shaped, and unimodal. The various aspects of this Bayesian analysis are discussed in great detail in Refs. $[10,17]$.

The best-fit analysis described above delivered $k=1$ or 2, depending on the $Q$ value. Figure 2 compares IXS spectra measured in pure glycerol and in the 200-nm Au-Np suspension at a few representative $Q$ values with the respective best-fitting line shapes and the individual spectral components introduced in Eqs. (2) and (3); equivalent curves for the 50-nm Au-Np suspension are shown in Fig. $2 \mathrm{~S}$ of the Supplemental Material. Again, the IXS spectral shapes of glycerol are those previously measured in Ref. [11].

Overall, both Figs. 1 and 2 evidence that the highfrequency inelastic modes in glycerol spectra persist in the $\mathrm{Np}$ suspension ones, yet with a strongly reduced relative amplitude. The comparison in Fig. 2 of experimental and model line shapes in the two samples further stresses the emergence of an additional low-frequency mode in the $\mathrm{Au}-\mathrm{Np}$ suspension spectrum whose origin is still mostly unclear, and having the effect to produce the broadening of the central peak mentioned before. A more precise assignment of this lowenergy mode, hereafter referred to as $\mathrm{DHO}_{1}$, can be gained from Fig. 3, where the best-fit values of its inelastic shift $\left(\Omega_{1}\right)$ is compared to that $\left(\Omega_{2}\right)$ of the high-energy mode, $\mathrm{DHO}_{2}$. Current results are therein compared with previous IXS data on pure glycerol [18], and with both the hydrodynamic linear dispersion $\hbar c_{s} Q$ and its elastic counterpart $\hbar c_{\infty} Q$, where $c_{s}=$ $1920 \mathrm{~m} / \mathrm{s}$ and $c_{\infty}=3100 \mathrm{~m} / \mathrm{s}$ are the adiabatic and the elastic value of the sound velocity in glycerol, respectively derived from Refs. [19,20].

Current pure glycerol data displayed in Fig. 3 are obtained from reanalyzing, with the discussed Bayesian approach, previous IXS measurements in Ref. [11]. Results on the 50-nm $\mathrm{Au}-\mathrm{Np}$ suspension are also included for comparison, as measured either in this experiment (see Supplemental Material) or in our previous lower resolution IXS measurements in [8].

Overall, data in Fig. 3 exhibit a few clear trends discussed below in some detail.

a. The high-energy branch. This dispersive branch has been thoroughly investigated in literature and it is commonly assigned to a collective acousticlike longitudinal excitation of glycerol. At low and moderate $Q$ 's, the frequency of this

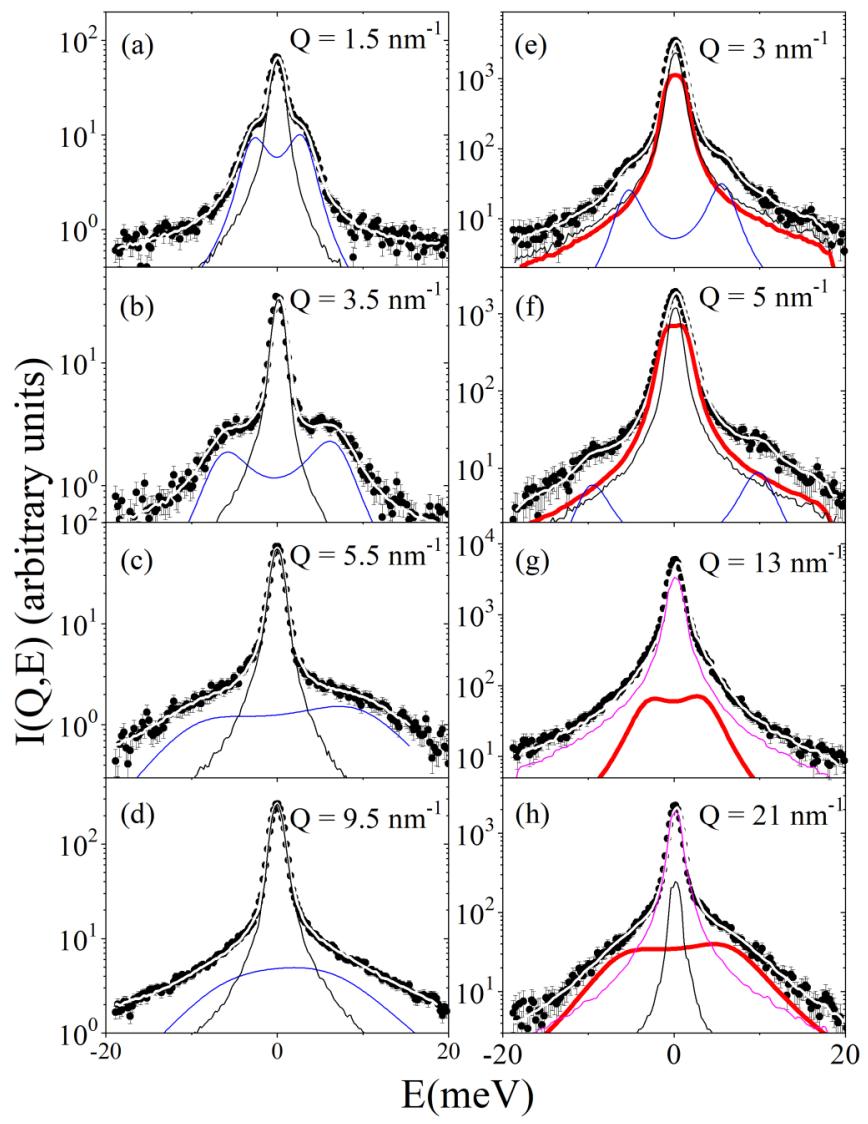

FIG. 2. (a)-(d) Representative IXS spectra of pure glycerol measured at some representative $Q$ 's (dots) are compared with the bestfitting model line shapes (white lines) along with its elastic (black line) and the inelastic DHO profile (blue line). (e)-(h) The same as in the left panel but referring to the 200-nm-diameter $\mathrm{Au}-\mathrm{Np}$ suspension in glycerol. Here the thick red and magenta lines represent the additional low-frequency excitation and the quasielastic Lorentzian contribution to the spectra, respectively.

mode, $\Omega_{2}$, largely exceeds the linear hydrodynamic dispersion, closely approaching the elastic one $c_{\infty} Q$. This is due to the circumstance that, in the $Q$ window probed by the current measurement, we prove the elastic response of the system. Also, the comparison between $\Omega_{2}$ 's in pure glycerol and in the suspensions suggests that, at this low concentration, immersed $\mathrm{Au}-\mathrm{Np}$ have little or no influence on the propagation of highfrequency density fluctuations.

At higher $Q$ values, $\Omega_{2}$ bends downward from the linear law $c_{\infty} Q$ due to the coupling of sound propagation with first-neighbor molecular ordering. Finally, at even higher $Q$ 's, $\Omega_{2}$ can no longer be determined reliably due to the gradual disappearance of the $\mathrm{DHO}_{2}$ profile (see, e.g., Fig. 2), for both pure glycerol [18] and the suspension.

We thus resorted to a different method to achieve an at least approximate determination of the high-frequency dispersive branch. As often proposed for simpler fluids, we estimated it through the maxima positions, $\Omega$, of the function $(E / Q)^{2} I(Q, E)$, assumed to be an "experimental" analog of the spectrum of the longitudinal current autocorrelation $(E / Q)^{2} S(Q, E)$. Here, $I(Q, E)$ represents the measured signal after subtraction of the background intensity. Some of these 


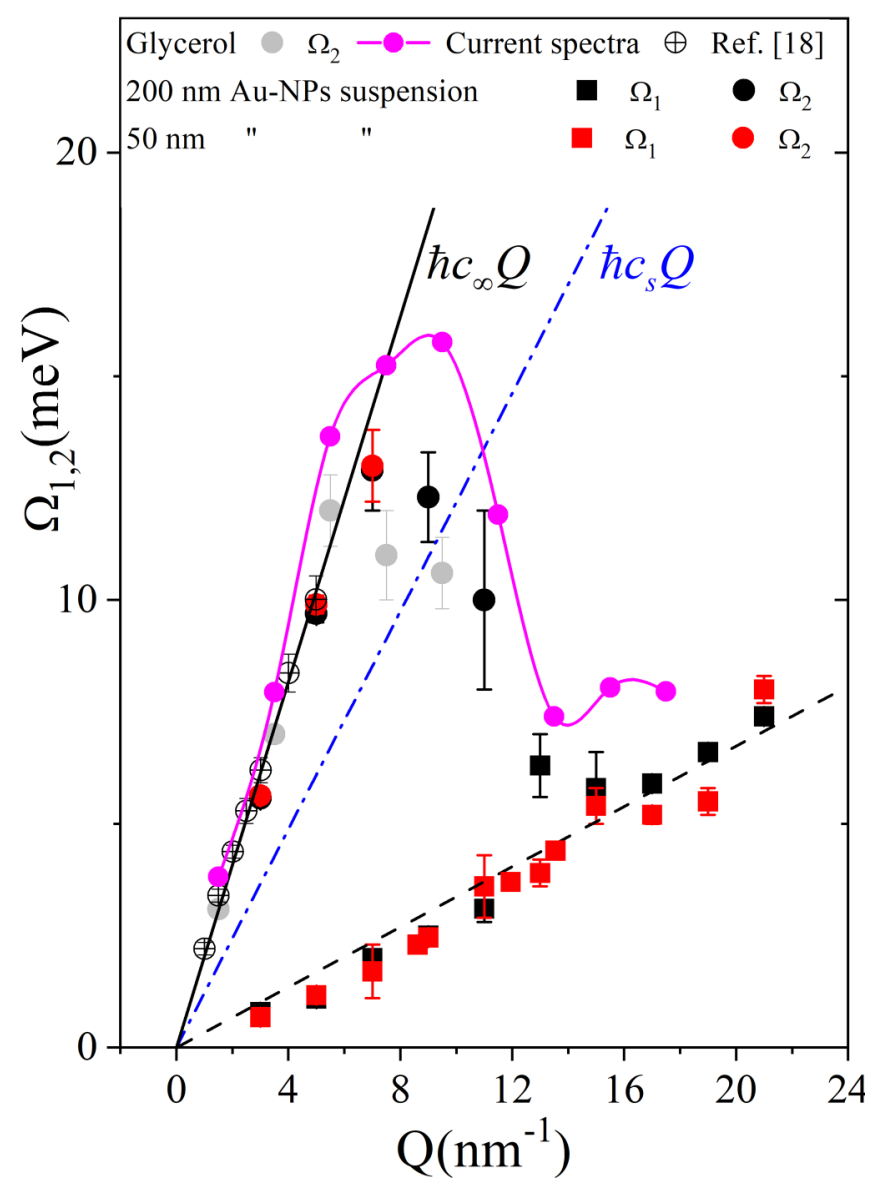

FIG. 3. Fitted values of low $\left(\Omega_{1}\right)$ and high $\left(\Omega_{2}\right)$ excitation energies are reported as a function of $Q$, the meaning of various symbols being specified in the legend. The $50-\mathrm{nm} \Omega_{1}$ curve (red squares) also include values obtained from the analysis of spectra measured on the Sector 3 beam line of APS with the same experimental conditions of the present experiment at $Q=8.52,11.95$, and $13.55 \mathrm{~nm}^{-1}$. The hydrodynamic linear dispersion of glycerol (blue dash-dotted line) and its "infinite frequency" counterpart (solid black line) having velocities of 1920 and $3100 \mathrm{~m} / \mathrm{s}$, respectively, are also shown for reference. The dashed black line represents the linear best fit of all $\Omega_{1}$ values, yielding a velocity of $520 \mathrm{~m} / \mathrm{s}$. Gray dots are obtained by applying the Bayesian analysis to IXS spectra of pure glycerol [11]. The maxima positions of the spectra of the longitudinal current autocorrelations, also derived from Ref. [11] for pure glycerol, are shown as magenta dots + spline. Finally, crossed circles represent the frequency of the DHO peaks obtained by Sette et al. for pure glycerol [18].

spectra are shown in Fig. S3 of the Supplemental Material, while $\Omega$ values estimated for glycerol are included as magenta dots+line in Fig. 3. Similar spectral functions obtained for the 50-nm Au-Np suspension are reported in Fig. S4 of the SM. This more extended determination of the dispersion shows a very typical trend, characterized by a bending downwards to a minimum close to the position of the first sharp diffraction maximum, i.e., about $14 \mathrm{~nm}^{-1}$. The circumstance that $\Omega_{2}$ values are consistent for the 50- and 200-nm suspensions likely is due to the large $Q d_{c}$ values considered in these measurements $\left(Q d_{c} \geqslant 150\right.$ and 600 for the two samples),

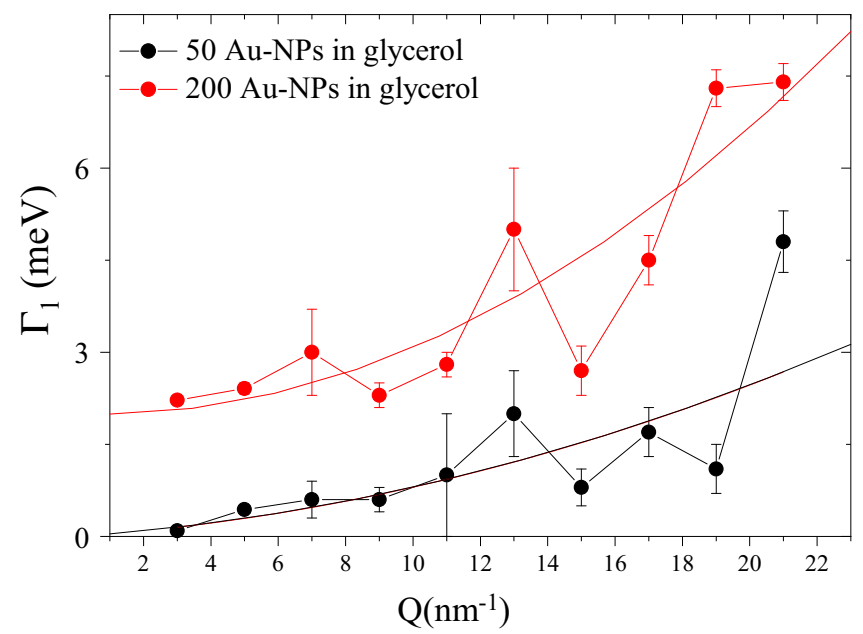

FIG. 4. Best-fit values of the low-energy mode half-width $\left(\Gamma_{1}\right)$ for the suspensions of $200 \mathrm{~nm}$ (red dots) and $50 \mathrm{~nm}$ (black dots) diameter $\mathrm{Au}-\mathrm{Np}$ are reported as function of $Q$ and compared with the respective parabolic fits (lines of corresponding colors) mainly serving as a guide to the eye. Results on the $50-\mathrm{nm} \mathrm{Au}-\mathrm{Np}$ suspension are vertically shifted for clarity.

which makes the Au-Np assimilable to huge bodies with infinite radius of curvature, i.e., essentially "rigid walls."

b. The low-energy branch. Although evidence for a lowenergy, $\mathrm{DHO}_{1}$, profile is found in all IXS spectra from the suspension sample, for $Q \leqslant 9 \mathrm{~nm}^{-1}$ its two peaks get too close to be adequately resolved by the measurement. Interestingly, best-fit values of $\Omega_{1}$ exhibit a nearly linear $Q$ increase across the whole $Q$ range and bear no evidence for a coupling with the local order, which primarily causes a minimum at the $Q$ position of the first diffraction peak. The mere existence of an additional low-energy mode in the suspension spectrum is interesting and deserves further comments. Although a second acoustic mode was observed by several quasimacroscopic measurements on suspensions of silica or plexiglas colloids [2,5,21], no evidence was documented at mesoscopic scales. Indeed, the only inelastic excitations thus far detected by IXS measurements on suspensions [8] are those either propagating throughout the liquid matrix or confined in the Np interiors.

The linear dispersion of $\Omega_{1}$ leads to estimate a propagation speed of about $(52 \pm 2) 10 \mathrm{~m} / \mathrm{s}$, i.e., much lower than the sound speeds of both glycerol and gold. This evidence could reveal the physical origin of such a mode, as the only excitation expected to propagate in a composite (liquid-solid) medium with a speed lower than the longitudinal sound velocities in both components is a Stoneley wave [22]. This is a propagating wave whose amplitude decays with increasing distance from the liquid-solid interface. It is worth noticing that the present findings extend previous BLS results $[2,4]$ into a $Q$ region well beyond the continuum limit and involving distances comparable with first-neighbor molecule separations.

Figure 4 illustrates the $Q$ dependence of $\Gamma_{1}$ for the 200and 50-nm Au-Np suspensions. As is often the case of IXS line-shape data analyses, the values have sizable scattering, as the determination of linewidths is usually less accurate than, for instance, $\Omega_{1}$ in Fig. 3. However, displayed values 
look compatible with a $Q^{2}$ growth (though they cannot rule out other functional forms), as shown by the comparison with parabolic best fits; this suggests the persistence of a hydrodynamiclike behavior in a remarkably extended $Q$ range, as already discussed for $\Omega_{1}$.

Although best-fitting parabolic curves in Fig. 4 are not exactly parallel, we attributed scarce significance to this difference. In fact, large random oscillation of $\Gamma_{1}(Q)$ data might unpredictably affect their best-fitting parabolas, also making the determination of actual $Q$ dependence uncertain.

As to the Lorentzian term in Eq. (2), its inclusion in the line-shape model rests on the evidence, for $Q \geqslant 7 \mathrm{~nm}^{-1}$, of a quasielastic component broader than the resolution profile. Its average unconvoluted HWHM, $z_{0} \sim 0.17 \mathrm{meV}$, is similar to that observed in two quasielastic neutron scattering measurements on glycerol [23,24], and also comparable with the one we estimated in the pure glycerol data only at the highest measured $Q=17.5 \mathrm{~nm}^{-1}\left(z_{0}=0.20 \pm 0.02 \mathrm{meV}\right)$. The reason why, for pure glycerol, this mode emerges at this $Q$ only is still unclear, even though it might be due to the more intense wings of the longitudinal acoustic shoulders partially hiding fine quasielastic features of the spectrum. Also, the observed behavior is not in contrast with the more popular $\delta(E)$ modeling of the central peak of pure glycerol IXS spectra at lower $Q$ 's $[18,20,25]$.

\section{CONCLUSIONS}

In conclusion, we discussed here the results of an inelastic x-ray scattering study of the terahertz spectrum of density fluctuations in a hybrid liquid-solid material such as a suspension of Au nanoparticles in glycerol. A joint Bayesian analysis of the current IXS measurements and previous ones in pure glycerol unambiguously demonstrates that the presence of an even small concentration of nanoparticles causes the emergence of an additional spectral mode, similar to the one observed by BLS measurements in the hydrodynamic regime, which we assign to Stoneley waves propagating at the Np-glycerol interface. Interestingly, this mode keeps an almost linear dispersive behavior down to mesoscopic scales without displaying $Q$ oscillations typically relating to local ordering, or any visible interactions with higher-energy acoustic modes. Overall, the present study probes the dynamic response of a suspension over distances comparable with nearest-neighbor atomic separations, thus ideally complementing previous spectroscopic measurements only covering continuous or quasimacroscopic scales [2-6]. Additional measurements of $\mathrm{Np}$ of various materials and shapes and dispersed in different liquid matrices will shed further insight into the intriguing spectral behaviors discussed in this paper.

\section{ACKNOWLEDGMENTS}

This work used resources of the National Synchrotron Light Source II, a U.S. Department of Energy (DOE) Office of Science User Facility operated for the DOE Office of Science by Brookhaven National Laboratory under Contract No. DE-SC0012704. This research was also funded by Ente Cassa di Risparmio Firenze (Grant No. 2016-0866) and by Ministero dell'Istruzione dell'Università e della Ricerca Italiano (Grant No. PRIN2017-2017Z55KCW).
[1] P. C. Martin, O. Parodi, and P. S. Pershan, Unified hydrodynamic theory for crystals, liquid crystals, and normal fluids, Phys. Rev. A 6, 2401 (1972).

[2] J. Liu, L. Ye, D. A. Weitz, and P. Sheng, Novel Acoustic Excitations in Suspensions of Hard-Sphere Colloids, Phys. Rev. Lett. 65, 2602 (1990).

[3] X. Jing, P. Sheng, and M. Zhou, Theory of Acoustic Excitations in Colloidal Suspensions, Phys. Rev. Lett. 66, 1240 (1991).

[4] L. Ye, J. Liu, P. Sheng, and D. A. Weitz, Sound propagation in suspensions of solid spheres, Phys. Rev. E 48, 2805 (1993).

[5] R. S. Penciu, G. Fytas, E. N. Economou, W. Steffen, and S. N. Yannopoulos, Acoustic Excitations in Suspensions of Soft Colloids, Phys. Rev. Lett. 85, 4622 (2000).

[6] R. Penciu, H. Kriegs, G. Petekidis, G. Fytas, and E. Economou, Phonons in colloidal systems, J. Chem. Phys. 118, 5224 (2003).

[7] R. Stoneley, Elastic waves at the surface of separation of two solids, Proc. R. Soc. London, Ser. A, Containing Papers of a Mathematical and Physical Character 106, 416 (1924).

[8] A. De Francesco, L. Scaccia, M. Maccarini, F. Formisano, Y. Zhang, O. Gang, D. Nykypanchuk, A. H. Said, B. M. Leu, A. Alatas et al., Damping off terahertz sound modes of a liquid upon immersion of nanoparticles, ACS Nano 12, 8867 (2018).

[9] A. De Francesco, L. Scaccia, F. Formisano, E. Guarini, U. Bafile, M. Maccarini, A. Alatas, Y. C. Cai, and A. Cunsolo, The terahertz dynamics of an aqueous nanoparticle suspension: An inelastic x-ray scattering study, Nanomaterials 10, 860 (2020).

[10] A. De Francesco, E. Guarini, U. Bafile, F. Formisano, and L. Scaccia, Bayesian approach to the analysis of neutron Brillouin scattering data on liquid metals, Phys. Rev. E 94, 023305 (2016).

[11] A. Cunsolo, B. M. Leu, A. H. Said, and Y. Q. Cai, Structural and microscopic relaxations in glycerol: An inelastic x-ray scattering study, J. Chem. Phys. 134, 184502 (2011).

[12] A. H. Said, H. Sinn, and R. Divan, New developments in fabrication of high-energy-resolution analyzers for inelastic $\mathrm{x}$ ray spectroscopy, J. Synchrotron Radiat. 18, 492 (2011).

[13] T. Toellner, A. Alatas, and A. Said, Six-reflection MeVmonochromator for synchrotron radiation, J. Synchrotron Radiat. 18, 605 (2011).

[14] See Supplemental Material at http://link.aps.org/supplemental/ 10.1103/PhysRevE.102.022601 for IXS spectra of 50-nmdiameter Au-Np, selected spectra at four different momentum transfer vectors $Q$ with correspondent best fits and line-shape analysis, experimental current spectra for pure glycerol, and 200-nm Au-Np suspension in glycerol.

[15] G. Monaco, A. Cunsolo, G. Ruocco, and F. Sette, Viscoelastic behavior of water in the terahertz-frequency range: An inelastic x-ray scattering study, Phys. Rev. E 60, 5505 (1999).

[16] A. Cunsolo, G. Ruocco, F. Sette, C. Masciovecchio, A. Mermet, G. Monaco, M. Sampoli, and R. Verbeni, Experimental 
Determination of the Structural Relaxation in Liquid Water, Phys. Rev. Lett. 82, 775 (1999).

[17] P. J. Green, Reversible jump Markov chain Monte Carlo computation and Bayesian model determination, Biometrika 82, 711 (1995).

[18] F. Sette, M. H. Krisch, C. Masciovecchio, G. Ruocco, and G. Monaco, Dynamics of glasses and glass-forming liquids studied by inelastic x-ray scattering, Science 280, 1550 (1998).

[19] M. Rajeswari and A. K. Raychaudhuri, Specific-heat measurements during cooling through the glass-transition region, Phys. Rev. B 47, 3036 (1993).

[20] A. Giugni and A. Cunsolo, Structural relaxation in the dynamics of glycerol: A joint visible, UV and x-ray inelastic scattering study, J. Phys.: Condens. Matter 18, 889 (2006).
[21] X. Jing, P. Sheng, and M. Zhou, Acoustic and electromagnetic quasimodes in dispersed random media, Phys. Rev. A 46, 6513 (1992).

[22] L. Brekhovskikh, Waves in Layered Media (Elsevier, New York, 2012), Vol. 16.

[23] D. Aranghel, V. Tripadus, A. Radulescu, M. Petre, M. Dima, and C. Petre, Quasielastic neutron scattering on glycerol, J. Optoelectron. Adv. Mater. 12, 953 (2010).

[24] A. Vispa, S. Busch, J. L. Tamarit, T. Unruh, F. FernandezAlonso, and L. C. Pardo, A robust comparison of dynamical scenarios in a glass-forming liquid, Phys. Chem. Chem. Phys. 18, 3975 (2016).

[25] G. Monaco and V. Giordano, Breakdown of the Debye approximation for the acoustic modes with nanometric wavelengths in glasses, Proc. Natl. Acad. Sci. USA 106, 3659 (2009). 